\title{
Intramural Recurrence Without Mucosal Lesions After an Endoscopic Mucosal Resection for Early Colorectal Cancer
}

\author{
Min Sung Kim, Nam Kyu Kim , Ji Hye Park ${ }^{2}$ \\ Department of Surgery, Eulji General Hospital, Eulji University School of Medicine, Seoul; Departments of ${ }^{1}$ Surgery and ${ }^{2}$ Pathology, Yonsei \\ University College of Medicine, Seoul, Korea
}

\begin{abstract}
Advances in endoscopic instruments and techniques have enabled increased detection and removal of early colorectal cancer (ECC), which is defined as a tumor whose invasion is limited to the mucosa or submucosa. Some cases can be treated by endoscopic mucosal resection (EMR). However, local recurrence frequently occurs after an EMR for ECC. The recurrence pattern is usually intramural recurrence with a mucosal lesion at the EMR's site. We report the cases of two patients with intramural recurrence without mucosal lesions after an EMR for ECC. These cases indicate that a local recurrence after an EMR for ECC can appear as an intramural recurrence without mucosal lesions at a previous EMR site or another site, although this presentation is very unusual.
\end{abstract}

Keywords: Colorectal neoplasms; Endoscopic mucosal resection; Local neoplasm recurrences; Local neoplasm recurrence

\section{INTRODUCTION}

The incidence of early colorectal cancer (ECC) has been increasing. Endoscopic mucosal resection (EMR) for ECC is being performed more frequently than in the past. Consequently, recurrent rectal cancer after an EMR has increased in frequency. However, few studies have reported the recurrence rate and the long-term outcome after an EMR for ECC. In a few studies, the local recurrences after EMRs for benign and malignant polyps were mostly intramural recurrences with a mucosal lesion at the EMR site [14]. In this report, two cases that show local intramural recurrence without a mucosal lesion after EMR are described. This pattern has rarely been reported in the published literature.

Received: February 14, 2013 - Accepted: April 25, 2013

Correspondence to: Nam Kyu Kim, M.D.

Department of Surgery, Severance Hospital, Yonsei University College of

Medicine, 50 Yonsei-ro, Seodaemun-gu, Seoul 120-752, Korea

Tel: +82-2-2228-2117, Fax: +82-2-313-8289

E-mail:namkyuk@yuhs.ac

(C) 2013 The Korean Society of Coloproctology

This is an open-access article distributed under the terms of the Creative Commons Attribution NonCommercial License (http://creativecommons.org/licenses/by-nc/3.0) which permits unrestricted non-

commercial use, distribution, and reproduction in any medium, provided the original work is properly cited.

\section{CASE REPORT}

\section{Case 1}

A 54-year-old Korean man underwent screening colonoscopy in May 2007. The colonoscopic examination revealed a $1.5-\mathrm{cm}$ sessile polyp in the rectosigmoid colon. The polyp was located $20 \mathrm{~cm}$ from the anal verge. An en bloc EMR was performed for this lesion. Histopathologic examination of the specimen showed a moderately-differentiated adenocarcinoma invading the submucosal layer (depth of invasion, $750 \mu \mathrm{m}$ ). There was no definite lymphovascular invasion, and the surgical margins were negative for cancer cells (length of deep resection margin, $0.7 \mathrm{~mm}$; length of deep resection margin, not clearly reported). The patient was referred to Severance Hospital, but additional surgical treatment was not performed.

During the 43 months after the EMR, routine surveillance was performed. After the 43 months, the patient's carcinoembryonic antigen (CEA) level was elevated $(5.10 \mathrm{ng} / \mathrm{mL})$. Examinations to exclude the possibility of recurrent rectosigmoid colon cancer, including computed tomography (CT) from the abdomen to the pelvis and 18-fluorodeoxy-glucose-positron emission tomography (FDG-PET), demonstrated local recurrent sigmoid colon cancer without distant metastasis. A colonoscopy showed an EMR scar without gross evidence of mucosal local recurrence (Fig. 1).

The patient underwent radical surgery. Examination of the mu- 


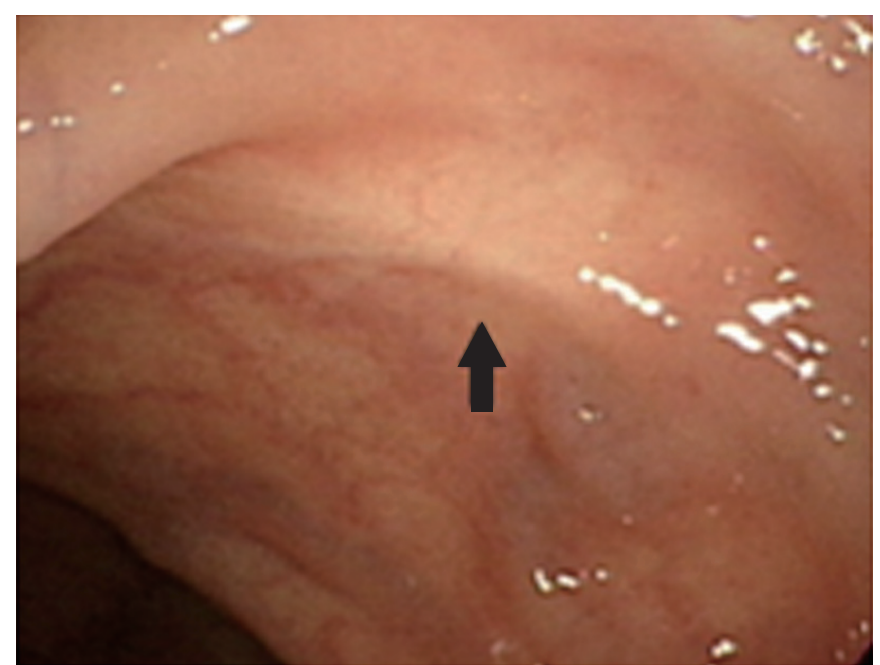

Fig. 1. Colonoscopy revealed the surface of the postendoscopic mucosal resection site, which shows a white scar (arrow) without gross evidence of an intraluminal local recurrence (case 1).

cosal surface of the specimen showed no specific lesion. Examination of the serosal surface revealed a firm, solid, mass-like lesion (7 $\mathrm{cm} \times 3 \mathrm{~cm}$ ) that was $10 \mathrm{~cm}$ from the proximal resection margin and $4 \mathrm{~cm}$ from the distal resection margin. Multiple sections showed a yellowish-tan mass located in the submucosal, muscular, and subserosal areas. Pathologic examination of the specimen showed a moderately-differentiated rpT4aN0 adenocarcinoma with lymphovascular and perineural invasion (Fig. 2). The postoperative course was uneventful and the patient was discharged on the tenth day.

\section{Case 2}

A 58-year-old Korean woman underwent screening colonoscopy in August 2006. Colonoscopic examination revealed a $1.5-\mathrm{cm}$ sessile polyp in the rectum. The polyp was located $9 \mathrm{~cm}$ from the anal verge, and a biopsy was performed. Pathological examination suggested the presence of an adenocarcinoma. The patient was referred to our hospital. Her CEA level was $1.5 \mathrm{ng} / \mathrm{mL}$. Abdominalpelvic CT and rectal magnetic resonance imaging (MRI) showed no evidence of significant focal lesions or distant metastases.

An en bloc EMR was performed for the lesion. Histopathologic examination of the specimen showed a moderately-differentiated adenocarcinoma that had invaded the submucosal layer (depth of invasion, $200 \mu \mathrm{m}$ ) with lymphovascular invasion. The surgical margins were negative for cancer cells (length of deep resection margin, $0.4 \mathrm{~mm}$; length of deep resection margin, not clearly reported). At the patient's request, no additional surgical treatment was performed. Routine surveillance was performed for 56 months following the EMR. After 56 months, a routine surveillance PETCT of the abdomen and pelvis revealed a pelvic mass with abnormal hypermetabolism on the left side of the mesorectum. The lesion was highly suspicious for recurrence. Rectal MRI showed a

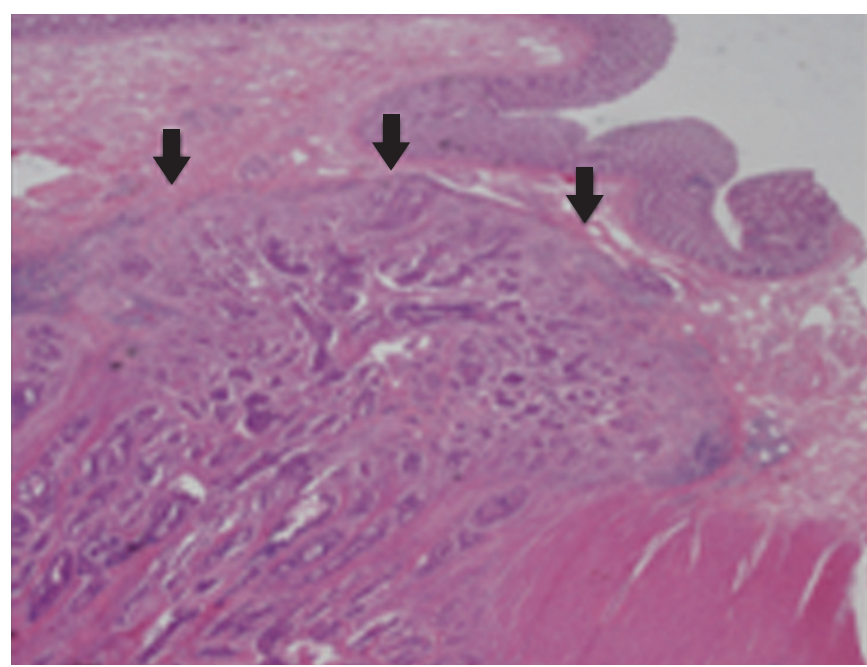

Fig. 2. Pathologic examination revealed a moderately-differentiated adenocarcinoma with lymphovascular and perineural invasion. In a previous endoscopic mucosal resection scar, the cancer was located in the submucosal, muscular, and subserosal areas, and grew exclusively in the submucosal, muscular, and subserosal layers without mucosal lesions (arrows) (case $1 ; \mathrm{H} \& \mathrm{E}, \times 12$ ).

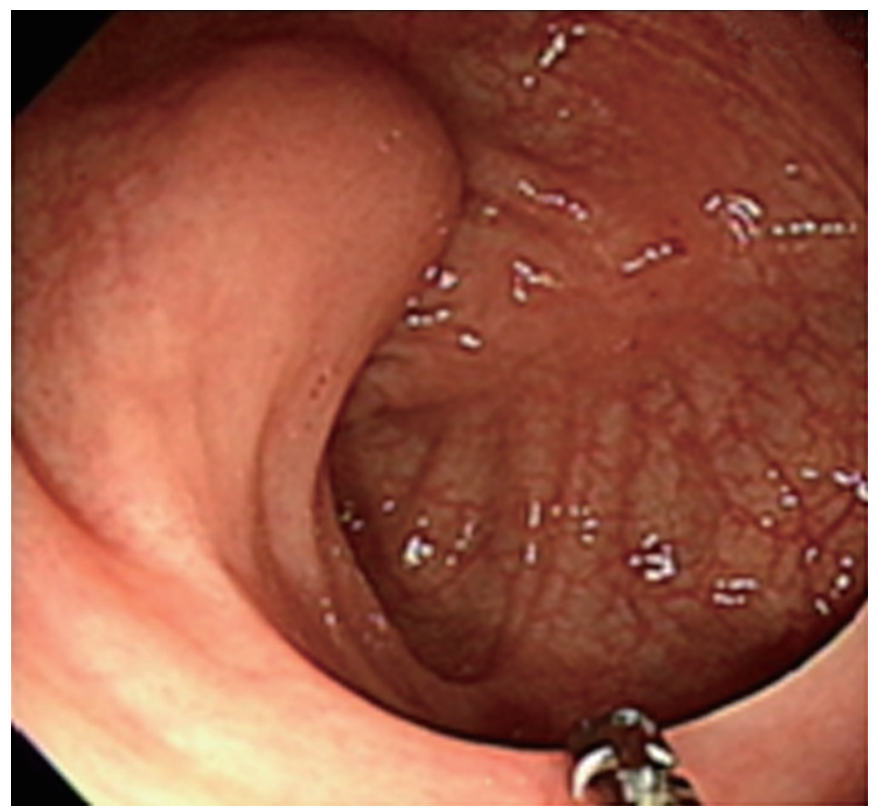

Fig. 3. Colonoscopy revealed a submucosal, 2-cm-diameter, tumorlike lesion in the rectum. The mucosal surface was soft and smooth, with no evidence of mucosal change. The lesion was seen around the post-endoscopic mucosal resection scar, $10 \mathrm{~cm}$ from the anal verge (case 2).

2-cm solid mass in the left side of the mesorectum with direct invasion into the left midrectal wall at $10 \mathrm{~cm}$ above the anal verge. By MRI, the lesion appeared to be a perinodal extension of a lymph-node metastasis. 


$\begin{aligned} \text { Annals of } & \begin{array}{l}\text { Intramural Recurrence Without Mucosal Lesions After an Endoscopic Mucosal Resection for Early } \\ \text { Colorectal Cancer }\end{array} \\ \text { Coloproctology } & \begin{aligned} \text { Min Sung Kim, et al. } \\$\cline { 2 - 3 }\end{aligned}\end{aligned}
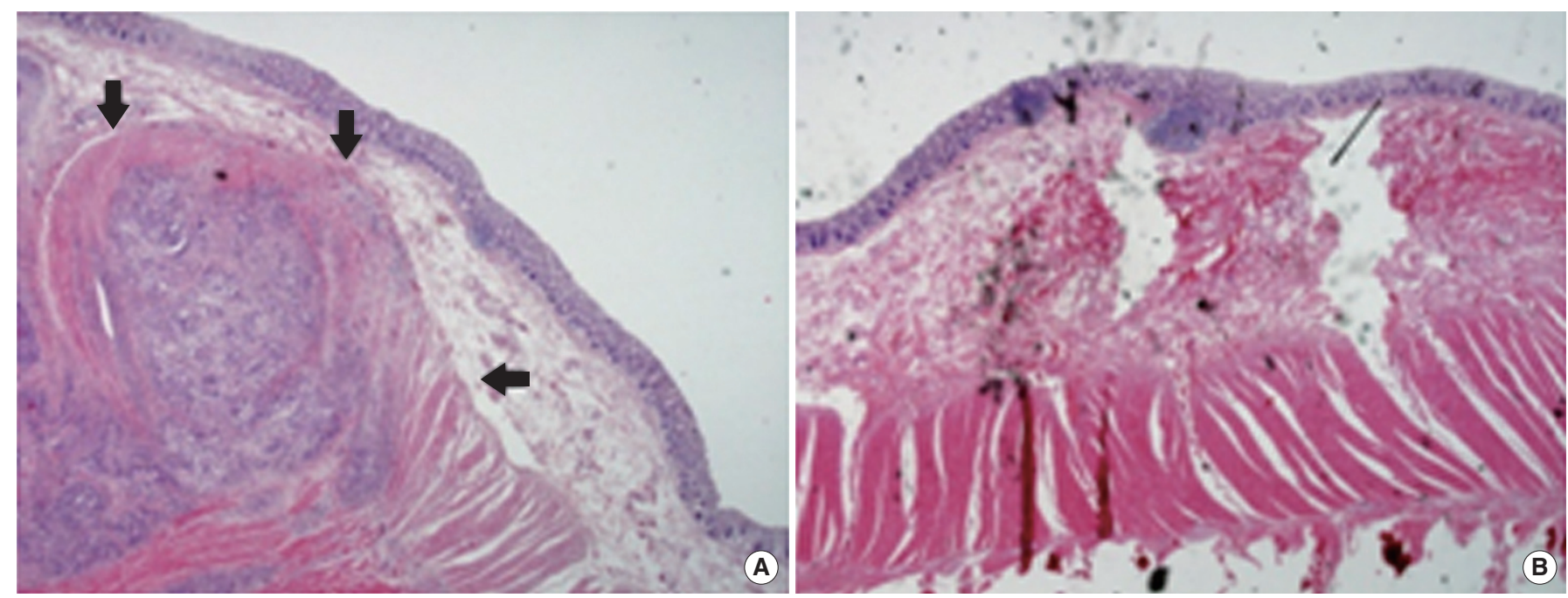

Fig. 4. (A) Pathologic examination demonstrated a $1.5-\mathrm{cm}$, moderately-differentiated adenocarcinoma adjacent to the endoscopic mucosal resection (EMR) scar that was located in the submucosa, muscle, and perirectal fat tissues with no evidence of mucosal change (arrows) (case 2; $\mathrm{H} \& \mathrm{E}, \times 12$ ). (B) No residual cancer was seen on pathologic examination of the previous EMR scar (case 2; H\&E, $\times 12$ ).

Colonoscopy revealed a submucosal, tumor-like lesion approximately $2 \mathrm{~cm}$ in diameter in the rectum (Fig. 3). The lesion was near the post-EMR scar, $10 \mathrm{~cm}$ from the anal verge. The patient underwent radical surgery. Examination of the mucosal surface of the specimen showed a $1.5-\mathrm{cm}$ submucosal mass adjacent to the EMR scar with no evidence of mucosal change. Multiple sections showed a yellowish-tan mass in the submucosal, muscular, and perirectal fat tissues. Pathologic examination of the specimen showed a moderately-differentiated $\mathrm{rpT} 3 \mathrm{~N} 1 \mathrm{~b}$ adenocarcinoma with lymphovascular and perineural invasion. However, no residual cancer was seen at the EMR scar (Fig. 4). The resection margin showed no sign of the adenocarcinoma. The postoperative course was uneventful, and the patient was discharged on the seventh day.

\section{DISCUSSION}

Intramucosal cancer can be cured by using a complete EMR, as there is no risk of lymph-node metastasis [5]. However, submucosal cancer shows lymph-node metastasis in 6-12\% of the cases [6]. The management of submucosal cancer remains controversial, with various opinions on the treatment policy for ECC patients after endoscopic removal. One opinion is that all patients with polyps containing an invasive carcinoma should undergo a standard resection. Another is that a conservative approach should be maintained in the absence of cancer at the resection line. For cases of submucosal cancer with lymph-node metastasis, radical surgery with a lymphadenectomy is necessary. However, for cases without lymph-node metastasis, an endoscopic resection may be the most appropriate therapy. The key to deciding whether a complete EMR is adequate for the treatment of submucosal cancer is to determine the risk of lymph-node metastasis or local recurrence. Therefore, predicting lymph-node metastasis is critical. Many studies have investigated the clinical and the histological factors that predict lymph-node metastasis. Many have demonstrated that lymph-node metastasis is closely associated with the depth of submucosal invasion (SM depth) and with histologic factors such as lymphovascular invasion, poor differentiation, positive resection margin, and tumor budding. Of the factors generally assumed to be predictive of lymph-node metastasis, the SM depth is one of the most important [5, 7].

Submucosal cancer can be stratified as "low risk" and "high risk" based on favorable and unfavorable histologic criteria, respectively. In general, the mainstream opinion is that an endoscopic resection alone can be considered adequate for submucosal cancer in the low-risk category, but a curative surgical resection is recommended for submucosal cancer in the high-risk category with unfavorable histology involving lymphovascular invasion, poor differentiation, deep submucosal invasion $(\geq 1,000 \mu \mathrm{m})$ or positive resection margin.

Local recurrence frequently occurs after an EMR for large sessile tumors. Although comparing different studies is difficult because of the wide variations in the polyp size and the length of followup, the rate of recurrence at the polypectomy site after EMR for large sessile tumors is reported to be $10-46 \%[1,3,8,9]$. However, few studies have reported the recurrence rate after an EMR for ECC, and the long-term outcomes of an EMR for ERC have yet to be established. The recurrence rate of malignant colorectal polyps after EMR varies among the studies. Kikuchi et al. [10] assessed the risk of recurrence in 17 patients with early-stage colorectal cancer that had infiltrated the upper third of the submucosa (SM1). After an EMR, no local recurrence or lymph-node metastasis was 
observed to have occurred in any of the patients. Seo et al. [2] showed that the recurrence rate was 3.1\% for benign polyps and $33.3 \%$ for malignant polyps. In other words, an EMR of malignant polyps resulted in a higher recurrence rate than an EMR of benign polyps. In contrast, at a median follow-up of 12.3 months, Conio et al. [4] reported a similar recurrence rate of $21.4 \%$ for benign polyps and $24.3 \%$ for malignant polyps with favorable histopathology.

We think that 2 possible pathways for local recurrence are insufficient excision and micrometastatic nodal involvement. Insufficient excision could result from microscopic residual cancer cells implanting themselves into the unhealed area following the EMR. In the studies mentioned above, local recurrences after an EMR for large sessile tumors almost always occurred at the EMR site. This recurrent pattern might be due to microscopic residual cancer cells. We think that, in our first case, the origin of the local recurrence of exfoliated cancer cells being their implanting themselves into the unhealed area with subsequent healing of the overlying mucosa is highly likely. However, no recurrent lesion was found at the EMR site in our second case. Intramural recurrence without mucosal change occurred in the other rectal wall. Therefore, we believe that an insufficient excision is a highly-unlikely explanation for the local recurrence in our second case. The best possible explanation for the second case is a disseminated event caused by lymphatic seeding from hidden nodal involvement or the extensive lymphovascular permeation observed in the previous EMR finding.

The detection rate of ECC or colorectal polyps is rapidly increasing; consequently, EMRs are being performed at a higher rate. As the EMR becomes more frequent, a recurrent pattern such as that observed in our two cases should occur. Therefore, clinicians must remain alert for intramural recurrence without mucosal lesions in follow-up colonoscopies. Endoscopic examinations were scheduled as follows in these two cases: the first endoscopic follow-up was performed 3 months after the resection for submucosal cancer; additional endoscopic examinations were performed at 6-month intervals for the first 2 years after resection.

Aggressive surveillance after an EMR is essential because of the high risk of recurrence. After an EMR, follow-up should include regular endoscopic surveillance of the rectum and of the scar in particular. Careful follow-up to diagnose local recurrence early is necessary so that salvage surgery can be performed. If observations of this recurrent pattern increase, follow-up protocols should include endorectal ultrasonography that allows imaging of perirectal tissues. In the future, this recurrent pattern should be con- sidered when developing follow-up strategies.

\section{CONFLICT OF INTEREST}

No potential conflict of interest relevant to this article was reported.

\section{REFERENCES}

1. Tanaka S, Haruma K, Oka S, Takahashi R, Kunihiro M, Kitadai Y, et al. Clinicopathologic features and endoscopic treatment of superficially spreading colorectal neoplasms larger than $20 \mathrm{~mm}$. Gastrointest Endosc 2001;54:62-6.

2. Seo GJ, Sohn DK, Han KS, Hong CW, Kim BC, Park JW, et al. Recurrence after endoscopic piecemeal mucosal resection for large sessile colorectal polyps. World J Gastroenterol 2010;16:2806-11.

3. Saito Y, Fukuzawa M, Matsuda T, Fukunaga S, Sakamoto T, Uraoka T, et al. Clinical outcome of endoscopic submucosal dissection versus endoscopic mucosal resection of large colorectal tumors as determined by curative resection. Surg Endosc 2010;24:343-52.

4. Conio M, Repici A, Demarquay JF, Blanchi S, Dumas R, Filiberti $\mathrm{R}$, et al. EMR of large sessile colorectal polyps. Gastrointest Endosc 2004:60:234-41.

5. Cunningham KN, Mills LR, Schuman BM, Mwakyusa DH. Longterm prognosis of well-differentiated adenocarcinoma in endoscopically removed colorectal adenomas. Dig Dis Sci 1994;39: 2034-7.

6. Cooper HS, Deppisch LM, Gourley WK, Kahn EI, Lev R, Manley $\mathrm{PN}$, et al. Endoscopically removed malignant colorectal polyps: clinicopathologic correlations. Gastroenterology 1995;108:1657-65.

7. Hassan C, Zullo A, Risio M, Rossini FP, Morini S. Histologic risk factors and clinical outcome in colorectal malignant polyp: a pooled-data analysis. Dis Colon Rectum 2005;48:1588-96.

8. Hurlstone DP, Sanders DS, Cross SS, Adam I, Shorthouse AJ, Brown S, et al. Colonoscopic resection of lateral spreading tumours: a prospective analysis of endoscopic mucosal resection. Gut 2004;53:1334-9.

9. Higaki S, Hashimoto S, Harada K, Nohara H, Saito Y, Gondo T, et al. Long-term follow-up of large flat colorectal tumors resected endoscopically. Endoscopy 2003;35:845-9.

10. Kikuchi R, Takano M, Takagi K, Fujimoto N, Nozaki R, Fujiyoshi $\mathrm{T}$, et al. Management of early invasive colorectal cancer. Risk of recurrence and clinical guidelines. Dis Colon Rectum 1995;38: 1286-95. 